Voix et Images

voixetimages

\title{
Jean Éthier-Blais. Écrire ici
}

\section{Jean-Pierre Duquette}

Volume 2, numéro 3, avril 1977

Jean Éthier-Blais

URI : https://id.erudit.org/iderudit/200066ar

DOI : https://doi.org/10.7202/200066ar

Aller au sommaire du numéro

Éditeur(s)

Les Presses de l'Université du Québec

\section{ISSN}

0318-9201 (imprimé)

1705-933X (numérique)

Découvrir la revue

\section{Citer ce document}

Duquette, J.-P. (1977). Jean Éthier-Blais. Écrire ici. Voix et Images, 2(3), 307-319.

https://doi.org/10.7202/200066ar d'utilisation que vous pouvez consulter en ligne.

https://apropos.erudit.org/fr/usagers/politique-dutilisation/ 


\section{Jean Éthier-Blais}

Écrire ici

J.-P. D. Jean Éthier-Blais, vous êtes professeur, conférencier, romancier et essayiste. Mais on pense d'abord à vous comme au critique du Devoir: quinze années de chroniques hebdomadaires, cela signifie la lecture de quelques milliers de livres. Je voudrais vous demander tout de suite ce qui fait courir un liseur professionnel. Qu'avez-vous cherché au fil de ces pages innombrables et diverses? Et... qu'avez-vous trouvé? Comment envisagez-vous la critique littéraire? pourquoi la critique? en particulier la critique «immédiate», le billet hebdomadaire?

J. É.-B. Je pars du principe que la critique littéraire est un genre en soi. Un genre? C'est-à-dire une écriture qui permet de s'exprimer tout entier à l'écrivain qui a choisi d'en faire son instrument. Les plus grands artistes ont fait de la critique, qu'il s'agisse de Racine ou de Corneille dans leurs Prefaces, de Victor Hugo dans les siennes, de Lamartine (qui avait une sensibilité d'historien) ou d'Apollinaire. Je ne connais aucun créateur qui n'ait voulu s'expliquer sur le mode critique. Jusqu'à Aristophane qui donne dans le mode critique en se moquant de celle de son temps. Hertel disait que l'esprit critique tue le génie. Oui, s'il est démesuré, comme son absence totale fausse l'instinct créateur. C'est dire que, dans l'écriture, tout se tient. $A$ ceci, j'ajoute que la critique est un genre autonome. Qui, aujourd'hui, lit Saint-Marc Girardin ou Sophie Gay? Ils ne survivent que parce qu'un critique de génie a daigné parler d'eux. Sainte-Beuve fait revivre les personnages, faute de pouvoir animer leurs cuvres. C'est beaucoup plus souvent qu'on ne le pense, le cas. Le tort de nombreux critiques est de croire qu'ils sont uniquement au service des cuvres et des auteurs. Ils le sont, en effet, lorsqu'il s'agit d'une œuvre dont ils estiment qu'elle perdurera: opus aere perennius. Dans le domaine québécois, on lit Camille Roy ou Albert Lévesque, non pas Jeanne L'Archevêque-Duguay ou Rosaire Dion. $\mathrm{Ne}$ serait-ce pas que l'esprit critique, dès lors qu'il se situe dans une percée historique, s'épanouit singulièrement et devient la pierre de touche de. la qualité de la vie littéraire? Encore faut-il que le critique brise ses gonds, encastrés dans une réalité autre que la sienne propre. Sa lecture, processus ininterrompu, doit devenir le reflet de son âme et de 
sa vie. A cet égard, Sainte-Beuve, Gide ou Charles du Bos sont exemplaires. Ils durent parce qu'en eux le jugement a servi de décapant et que le critique a révélé, révèle chaque jour, l'homme.

Au besoin d'expression de soi le plus dense qu'il se peut, s'ajoutera l'amour des lettres. Le critique est d'abord un liseur. II est vorace. Tout lui est bon. Je note parfois les titres des livres qui sont sur ma table de nuit, à terre ou près de mon fauteuil. Aujourd'hui, 15 avril 1976, l'ldiot, la Tragédie du Général de Jean-Raymond Tournoux, Cet excellent Monsieur Danton, de Jacques Hérissay, les Enfantômes de Réjean Ducharme, le Coran, Au signe de Flore de Maurras, la Quête du Graal de Béguin et Bonnefoy. Mon âme liseuse veut tout connaître. Dans mon journal, parfois chaque jour sur une longue durée, je note ce qui, dans un livre, m'a frappé. Ce n'est pas un dialogue entre Tournoux et moi, mais une conversation au cours de laquelle Danton et les chevaliers de la Toison d'or partent, chacun de son côté, à la recherche de quelque ineffable. Lorsqu'il s'agit d'écrire un article, je cherche à rassembler quantité d'éléments épars qui donneront à mon jugement épaisseur et clarté. Le moment venu de parler d'un livre, de liseur je deviens lecteur. L'esprit en éveil, devant la page blanche, il s'agit d'une passe d'armes, d'une explication où je suis moins poreux. Car, en dépit du fait que je suis critique, c'est aussi pour moi que l'auteur a écrit son livre.

Quelles sont mes normes critiques?

1. Je crois d'abord à l'unité interne d'une cuvre et c'est vers elle que je vais d'instinct. Le style et la pensée doivent être ensemble comme, dans I'absolu, Don Quichotte et Dulcinée. Cette unité est indescriptible. On la sent, comme un chirurgien qui vous palpe sait, de science certaine, où se loge le kyste qui vous fera mourir. Certains écrivains ont la chance de naître portant en eux cette qualité d'unité: Pascal, Montherlant. On l'a, il faut remercier le ciel de ses largesses; on ne l'a pas, il faut tendre vers elle. Les écrivains simples la possèdent plus volontiers que les complexes. Cette simplicité est fille d'une parfaite connaissance des règles. L'art d'écrire, comme tout ce qui touche intimement à la vie des humains, est le résultat de plusieurs siècles d'analyses, de retours sur soi, d'échecs, de choix. Les écrivains qui durent et donc qu'on lit, sont ceux qui s'insèrent par la méditation et le travail dans cette recherche esthétique. L'écriture est un art. Elle n'est jamais ébauche, premier jet, satisfaction du besoin de se donner et de tout dire. L'unité du style, depuis toujours, on la trouve dans une œuvre taillée. Comme un arbre.

2. Dans toute cuvre, l'intelligence. Inutile de s'étendre là-dessus, I'intelligence existe et sait faire sentir sa présence. Peu m'importe que je sois d'accord ou non avec les propos d'un auteur. Chacun a le droit de penser comme il l'entend. II ne s'agit pas de penser «bien», mais de bien ordonner sa pensée. Le critique, dans ce domaine, est le maître du relatif. 
3. L'humanité. Tout romancier doit aimer ses personnages, vivre avec eux, avec eux souffrir et être heureux. Les aimer, c'est surtout leur donner la vie. L'abbé Herrera vit comme vit David Copperfield, dans son paysage et avec sa dimension. On rencontre des gens déplaisants. Ils n'en respirent pas moins. L'humanité du romancier consiste à voir les hommes tels qu'ils sont. C'est ce réalisme qu'on appelait autrefois la vraie humilité, qui est de savoir s'incliner devant les nuances de la vie.

4. J'aime, sur la page écrite, retrouver la pulsation du langage dans sa richesse. Que cette vie se traduise par la sécheresse d'une expression parfaitement tenue en laisse ou par le débordement d'un vocabulaire qui a rompu ses amarres, peu m'importe. Ce qui compte, c'est la certitude de se trouver en présence d'un écrivain en possession de ses moyens d'expression. Je reconnais que le français est une langue riche et difficile. Est écrivain celui qui tente, à chaque livre, de conquérir cet Himalaya. A quatre-vingts ans, Goethe déplorait son ignorance de l'allemand. Bienheureuse ignorance, qui naît de la connaissance profonde des choses.

5. Le sens des temps forts qui donnent à un livre son armature.

6. Chez les penseurs, la pensée.

7. Chez les poètes, le mystère dans une clarté supposée, ce compagnonnage inséparable de l'invention verbale et de la métaphysique. Je ne puis supporter une poésie d'où le sens est absent. Qu'est-ce donc qu'une suite incohérente de mots? Je suis un lecteur du dictionnaire, triomphe insurpassable dans ce genre d'incohérence, puisque les mots n'y ont aucun rapport entre eux. Les nomenclatures ne sont poétiques que par hasard. Je ne puis fréquenter que les poètes qui me forcent à m'intégrer à leur sentiment, soit qu'agisse sur moi la perfection de la musique, soit que m'emporte la puissance de l'image chantée.

8. Je lis peu les philosophes, trouvant ailleurs ma pâture. Exception, Nietzsche, qui m'émeut et m'éblouit toujours, dont je partage la conception du monde et de la littérature. Il a vécu dans un univers d'idées et de travail. Je jalouse profondément un homme qui a su devenir ce que je ne serai jamais.

9. Enfin, ce que je recherche avant tout dans la lecture, c'est, au niveau le plus élevé, qui est celui de Pascal ou de Benjamin Constant, le bonheur sans égal d'un style et d'une pensée qui ne font qu'un dans la lumière de la raison et du sentiment. Comment parler de ces écrivains sinon dans le contexte d'une expérience qui touche à la mystique? Dieu ici ne fait pas que parler au cœur, il écrit en lui.

J.-P. D. A côté de vos recueils d'articles critiques (la série des Signets), vous avez publié des poèmes, Asies; un roman: Mater Europa; des nouvelles: le Manteau de Rubén Darío. Comment êtes-vous passé de la critique à la création? 
J. É.-B. Un jour de l'été 1965, j'étais à Ottawa, chez Ludovic Hudon. J'avais à cette époque une passion pour les quatuors que Mozart a dédiés à Haydn, en particulier pour celui qu'on appelle "la Chasse." Dans les accents et les heurts en apparence désordonnés de cette musique, je croyais trouver un remède à mes peines insignifiantes. J'avais donc écouté “la Chasse" plutôt deux fois qu'une. Soudain, sans y penser, j'ai pris un papier et une plume et j'ai écrit la fin de la première nouvelle de Mater Europa. Cela est venu du tréfonds de mon être, comme dicté par la vie: le choix des mots, le rythme, l'angoisse de l'homme seul qui s'éveille dans la nuit noire, les pas dans l'escalier, la présence au loin de la mère qu'on ne reverra plus jamais, tout m'est venu, en cinq minutes, j'entends encore comme si j'y étais, le bruit du métal sur le papier, je revois les mots qui se forment et, selon un mouvement et un ordre d'instinct, qui recouvrent la page. C'est ainsi que j'ai commencé à écrire autre chose que des articles critiques. La plupart des critiques écrivent d'abord des vers, des romans. L'instinct créateur, en eux, se transforme peu à peu en écriture réflexive. Car toute critique qui a un sens est d'abord introspection. J'ai vécu le processus contraire. A partir de ces quelques phrases «données", j'ai raconté l'enfance et la jeunesse d'un homme imaginaire que j'ai appelé Théodore Salandon. Un amour de jeunesse est devenu Wilma Saintongel. Pour le reste, imagination et vie entremêlées. Aucun de mes livres ne s'est bien vendu, ou vendu tout court. Mais parfois je rencontre quelqu'un qui me dit: "J'ai lu Mater Europa " et jusqu'en Bretagne. Lorsque j'ai commencé à écrire des nouvelles et des poèmes, je me suis donné vingt lecteurs. Hé bien! je les ai. Ils sont venus à moi. J'aimerais les connaître pour les inviter à dîner, tous ensemble. Nous parlerions de tout, sauf de littérature. Ce serait charmant. Au menu, un de ces poulets au beurre chers à Léon Daudet.

Dans l'univers de l'écriture, rien n'est épars. Tout, par le style, se tient. Dans une lettre de la fin de sa vie, Edith Sitwell raconte que tout comme un pianiste fait ses gammes en musique, elle avait fait les siennes en poésie. L'âge mûr venu, elle a dominé (ou presque, et encore) son instrument, qui est oreille, vie intérieure, don et rejet de soi par les mots. L'écriture est un art qui ne s'apprend que par le travail. Qu'est-ce donc que ce travail? C'est d'abord la lecture. II faut savoir lire, comme les abeilles savent butiner. Je ne crois pas à la jeunesse des cafés. Les écrivains de demain sont dans une chambre, assis à une table, et ils lisent. IIs méditent les leçons des maîtres. Au Québec, nous avons des milliers de jeunes écrivains, qui «font» leur livre. On les publie, tel est le système. Et puis, on n'entend plus jamais parler d'eux! Ce n'est pas tout que d'écrire dix ou vingt fois son premier livre. L'écrivain vrai avance, d'un livre à l'autre, précautionneusement, en exprimant chaque fois un peu plus, ou un peu moins, de son jus, selon. II s'insère dans une continuité. Encore faut-il qu'il sache à quelle famille il appartient, quels sont les esprits qui le soutiendront et l'alimenteront en cours de route. 
L'un des drames les plus poignants de notre histoire, c'est la disparition, depuis un siècle, de notre élite intellectuelle. On sait que le peuple du XIXe siècle lisait tout autant qu'aujourd'hui; les journaux de l'époque en témoignent, ainsi que la production littéraire. Mieux encore, car c'est là ce qui importe, les Canadiens français s'étaient, en moins d'un demisiècle après la Conquête, pourvus d'une élite intellectuelle qui faisait corps avec eux et les représentait sans bavures. Je ne citerai que trois noms, à des époques différentes et rapprochées: Philippe de Gaspé, François-Xavier Garneau et Hector Fabre. Vingt autres personnalités les entourent. Ces hommes avec leur voix personnelle, parlaient pour nous. Nous voulions vivre. Le libéralisme québécois, comme le conservatisme québécois, avaient acquis une dimension, un profil autochtones. La religion québécoise, dont on médit beaucoup, avait une immense dignité d'appoint. Ce n'était pas l'âge d'or, loin de là, mais c'était un milieu d'évolution raisonnée qui nous appartenait en propre. Aujourd'hui, nous sommes, en Amérique une masse indifférenciée; nos "penseurs" répètent, dans un langage la plupart du temps incompréhensible, les encycliques sociologiques de Harvard ou de Paris. Peu à peu, nous oublions la réalité du français. Notre clergé nous a trahis, nous n'avons plus la foi. À la poursuite d'une vie en apparence facile, nous avons donné le coup de pied de l'âne à nos origines, à nos ancêtres, à notre passé. Notre jeunesse ignore son histoire. Espérons que le sursaut d'énergie vitale dont la nation québécoise vient de donner l'exemple ne sera pas un baroud d'honneur. En portant au pouvoir un parti qui associe son devenir à celui de son indépendance, le Québec entre dans un univers historico-symbolique dont il ignore la géographie. Aurat-il le courage d'assumer un destin fondé sur le travail et non plus sur les récriminations? Au XIXe siècle, Nietzsche dénonçait la faillite de l'Allemagne et des Allemands. Que dirait-il s'il était québécois? Mais l'Allemagne wilhelmienne, pays de haute culture, pouvait donner le jour à un Nietzsche. Le Québec ne peut même pas susciter ses contestataires, sinon dans la cohue des places et des subventions. Tous les écrivains de ma génération se sont rangés. Les places! Ils ont crié jusqu'au jour où ils ont obtenu la situation à laquelie ils rêvaient sans trop y croire. Radio-Canada, l'Office national du film, les musées, les universités regorgent de nos Julien Sorel. Parfois, l'envie leur prend encore de gueuler, et cela donne le déjeuner ridicule de provincialisme de l'Académie Goncourt. Nos écrivains pensaient que leur hargne ferait peur aux Français assemblés comme elle avait fait trembler l'establishment canadien et que quelque Gallimard ou Grasset leur ouvrirait ses portes. II n'en a rien été, Dieu merci! A l'exception notoire d'Anne Hébert (et dans une certaine mesure de Marie-Claire Blais) l'insuccès de nos romanciers en France et dans le monde est devenu un sujet de blague partout où l'on parle français. C'est là notre drame. Nos écrivains ont peu à dire. II leur manque (moi hélas! y compris) ce dynamisme culturel qui fait entreprendre et mener à bien une grande œuvre. La matière est pourtant partout autour de nous. L'histoire d'une famille mont- 
réalaise de 1837 à 1914 ? C'est le sujet des Buddenbrooks. Ou un roman centré sur la vie et la mort d'un poète comme Saint-Denys Garneau ? Ce sont là des sujets immenses (exemples symboliques) qui devront être écrits (ou leurs semblables) si nous voulons qu'existe une littérature québécoise.

Dans ces conditions, comment et pour qui écrire? Je reviens à moi. Je crois que mes chroniques du Devoir ont (ou ont eu) un public souvent ravi d'entendre une voix qui ne donne pas dans le conformisme fascistegauchisant à la mode, mais qui s'exprime en fonction d'elle seule. J'alimente et j'instruis. Je communique ce que je sais. Des gens qui autrefois me vouaient aux gémonies me font dire qu'ils me lisent aujourd'hui avec plaisir. Mais ce n'est pas assez. Aurai-je le courage d'entreprendre cette grande œuvre que je propose aux autres? Quel courage il me faudrait! Si on songe à ce que représente le travail de Musil ou de Doderer, on hésite avant de se jeter dans la fournaise. En ce moment, je termine la sixième mouture de mon Borduas, essai sur son temps et ses amis. Après, nous verrons. Je suis rapide et lent à la fois. Ainsi, depuis sept ans, je travaille à un recueil de poèmes: le Prince-Dieu, image d'une certaine quête de l'âme. Je l'abandonne, le reprends, je déchire, je colle, je re-déchire, je jette le manuscrit au fond d'un tiroir. Un jour j'aurai assez de cette lutte avec l'ange et le poème sera là, le combat terminé faute de combattants. Pour me consoler dans cette bataille, j'écris d'autres poèmes, des petits poèmes presque en prose, à la fois tristes et moqueurs, où affleurent joies et peines de chaque jour. En vérité, ce qui se passe, c'est que j'ai peu à peu, et comme malgré moi, découvert que j'étais un écrivain. J'ai mis beaucoup de temps à accepter cette servitude, qui remplira mon vieil âge et y fera pousser des fleurs. Sur la paresse, triomphe d'une réalité supérieure, qui oblige à obéir celui qu'elle possède. Paul Bourget disait qu'aux époques de décadence, on préférait le mot à la ligne, la ligne à la page, la page au livre et le livre à l'œuvre. L'œuvre entière, c'est ça le style. C'est elle qui, dans cette forme inachevée et parachevée que lui confère la mort de l'écrivain, fait éclater le talent ou son absence. L'œuvre est le garant de la qualité de l'âme et l'image des livres de Bergotte, éclairés jusque dans la mort, est vraie. Si j'avais été bien guidé, j'aurais, dès l'âge de vingt ans, commencé une cuvre. Une ceuvre construite selon des normes classiques: poésie, roman, théâtre, pour terminer par, de nouveau et toujours la poésie. J'ai été diplomate, je suis devenu et resterai professeur, ne suis-je pas condamné à être critique d'abord? Ainsi, la vision architecturale du travail de ma vie a été faussée. C'est par un effort de volonté, issu du besoin que je ressentais avec force d'exploiter ce que je sentais en moi qui ressemblait à des pouvoirs d'expression, que j'ai écrit des vers et tenté de donner la vie à des personnages de mon cru. Ce qui aurait dû couler de source a été suscité. Sans doute est-ce pourquoi, dans tout ce que j'écris, la part autobiographique est-elle si importante. Avant d'écrire, j'ai vécu. 
J.-P. D. On a dit à sa parution que Mater Europa était un roman autobiographique, en partie tout au moins. Roman d'apprentissage, en quelque sorte. Quel est le sens profond de cette quête du héros, de ce périple du retour aux sources?

J. É.-B. J'ai essayé, de façon hélas! trop linéaire, d'exprimer ce besoin dans Mater Europa où j'ai utilisé ce que Pierre-Henri Simon a appelé la technique des temps forts. Chaque épisode est construit comme un sonnet et s'achève par une action qui est vision et prise de conscience. J'ai repris cette technique, en l'amplifiant, dans le Manteau de Rubén Darío. Mater Europa est un cri du ccur où j'ai voulu faire passer la sensibilité de mes trente ans. Mon enfance a été heureuse, mon adolescence, pure, ma jeunesse, intellectuelle. J'ai connu Montréal à l'époque de Hertel, des Compagnons de Saint-Laurent, du Pellan de la Nuit des Rois, de Borduas. On y sentait vivre un grand nombre d'intelligences. Jean-Marc Léger, d'Iberville Fortier, Adèle Lauzon, rayonnaient. Pour moi, qui arrivait du nord de I'Ontario, ce fut un enchantement. Je me trouvais en rapport avec des intelligences ouvertes, dans une ville qui me semblait être une nouvelle Athènes. Aujourd'hui, la jeunesse intellectuelle suit les modes de Paris, le nez à terre. Moins colonisés qu'on ne l'est en ce moment, nous voyions la France avec affection et détachement. Ce sont ces jeunes gens d'alors, Léger et Fortier, qui ont mis en place en 1950 le numéro spécial qu'Esprit consacra au Québec, qui nous fit prendre conscience, selon les principes d'une analyse historique qui s'appliquait pour la première fois à notre destin, de notre singularité. II y avait Cité libre que la carrière de ses rédacteurs dans le siècle a rendue presque célèbre. Cette génération venait des collèges classiques et donnait raison à leur enseignement en le dépassant.

Ensuite, il y eut la rue d'Ulm. Pendant la première année de mon séjour parisien, je me suis tu et j'ai laissé entrer librement en moi la musique de la langue française et ses cacophonies. Pendant deux ans, j'ai partagé la table de Raymond Weil, d'André Caquot, de Claude Taha-Hussein, de Jean Deshayes, de Roger Rémondon. J'ai beaucoup appris d'eux. Nous sommes restés des amis, tous universitaires, tous un peu poètes. J'étais heureux, même si j'ai toujours eu le bonheur triste. C'est aussi à Paris que j'ai connu les deux femmes qui ont le plus marqué ma vie: celle dont je garde le nom in pectore et Marie de Romanet, à qui je dédie tous mes Signets. J'ai vécu à Paris de 1948 à 1952, de 1954 à 1957, en 1968-1969; j'y ai passé I'hiver dernier, j'écris ceci à Strasbourg. Et tous ces merveilleux étés à la Messardière! En somme, près de dix ans de ma vie en France, à y accomplir mes racines québécoises. On n'est même pas à demi québécois lorsqu'on n'est pas, dans son cœur, français. II faut cette ouverture sur le monde, que l'Amérique ne peut nous donner. Je sais que c'est là affaire de tempérament et que la lutte larvée entre francophiles et francophobes au Québec est sans doute un riche ferment. Je me range parmi ceux qui ont l'honneur de 
connaître et d'aimer la France et qui croient, eux-mêmes d'origine française et n'ayant pas trahi leur langue, qu'en la servant, c'est aussi leur nation qu'ils servent et qu'en étant patriotes, c'est aussi indissolublement vers la civilisation française qu'ils se tournent. Ceci ne m'empêche en rien de houspiller les défauts des Français. Ils me sont aussi chers - et nécessaires - que les défauts de mes compatriotes. Je dois tout autant à la France qu'à mon propre pays. C'est une chance que j'ai eue, bénéfique, oui.

Pourtant, la. France ne figure pas dans Mater Europa. Par pudeur, j'ai supprimé de ce livre tout ce qui touchait à cette rencontre, pour moi, fondamentale; aussi parce que ma vie à Paris, si je la racontais, serait la matière d'un énorme récit, qui n'en finirait plus. Que d'expériences diverses, depuis mon régime d'étudiant jusqu'à la vie mensongère des ambassades. Et les pulsations, par-dessus tout cela, de la Ville! Rubén Darío complète Mater Europa. La notion d'exil y est partout présente, celle qui veut que l'homme soit sur cette terre, un étranger. Ni l'amour, ni la volupté de la connaissance, ni la réalisation des rêves les plus doux ne peuvent satisfaire ce qu'il y a en nous d'ineffable. Comme tous mes livres, j'ai longuement ruminé celui-là, qui est un bloc informe détaché de ma vie intérieure. Poésie et vérité, la formule de Goethe se retrouve partout et l'acte d'écrire, dans ses prolongements, est un dialogue entre ces deux mots. II m'a fallu trois ans de rejets et de reprises pour écrire la première nouvelle de ce recueil, nouvelle qui est presque un roman. Le style se ressent de ces atermoiements: manque d'unité d'écriture, épisodes comme plaqués sur le réel, personnages schématiques (sauf, peut-être, celui de Delloise, portrait de Roy Royal), dialogue souvent emprunté. Des trois nouvelles, celle qui me déplaît le moins, c'est la seconde, exercice proustien où chaque épisode se rapproche de ce qui est sans doute la vérité, sans jamais l'atteindre. C'est une nouvelle dont le labyrinthe est le personnage principal ; le labyrinthe et les découvertes qu'il présente à chaque tournant. Quant à la nouvelle qui donne son titre au recueil, elle souligne à quel point tout idéal est faux. Dans notre vie, tout est mensonge, à commencer par la vie. Rubén Darío découvre le vrai Verlaine, qui lui révèle le monde. Peu de gens peuvent accepter cette réalité. "La poésie, dit Verlaine, c'est de la merde." II a raison et pourtant, il a consacré sa vie à cette brenne. C'est que sans elle, il n'y a pas de poésie. Réconciliation des irréconciliables.

Et puis, dans ce livre, la nuit, les animaux, la terre mouillée, les sousbois, la musique, les prodiges, les regards, les amours, les citations cachées; un lecteur attentif se rendra vite compte que tout ce que j'écris est $v u$. Je raconte, j'essaie de capter un mouvement, une femme qui marche dans la rue, deux femmes dans un salon, une jeune homme au concert, peu importe, tout doit être dans la rétine du narrateur. II est un témoin privilégié. En sorte que dès que j'écris une nouvelle, je vis un curieux dédoublement; j'invente une action que je décris objectivement, comme si j'étais un autre. Je me vois écrivant, la plume à la 
main et dans les airs, comme le diable boiteux. Ce n'est pas que j'aime écrire. Au contraire. II le faut, pour les génies immenses comme pour les tâcherons. C'est dans' cette volonté d'accepter l'inévitable créateur que réside la communion des esprits.

Rien de ce que j'écris n'est «social» et mes personnages vivent dans un monde à part, régi par des problèmes d'ordre esthétique. Estelle est sauvée d'elle-même par l'appel d'un ordre supérieur et Rubén Darío voit s'écrouler à ses pieds, d'un seul mot, son idéal de perfection. L'un monte, l'autre descend, à l'intérieur d'un même univers. II faut être un Soljénitsyne pour s'intégrer dans le royaume politique ou militaire; un Thomas Mann pour récrire la Bible; ou Balzac. Le rêve de chaque écrivain est d'accéder à l'épopée et la loi des genres correspond à un besoin profond de l'homme. La stérilité intellectuelle qui, après la guerre, a donné naissance à la littérature française contemporaine trouve elle-même sa source dans cette dégradation des valeurs qui accompagne partout le socialisme, comme son Doppelgänger.

Jusqu'à présent, dans mes livres, je n'ai jamais parlé que de moi et de mes entours immédiats. C'est un tort. Mais je sens que cette époque de ma vie touche à son terme et que les poèmes que j'écris en ce moment marquent ce tournant. La lutte de Jacob et de l'ange symbolise ce combat de l'écrivain avec sa subjectivité. II faut bien quelque jour reconnaître que le monde extérieur a aussi une valeur et qu'en s'intégrant à lui par l'écriture, en l'animant, en l'obligeant à se dépasser, on se transforme et se grandit soi-même. Les carnets des écrivains sont intéressants, certes; les livres qui leur ont donné naissance sont plus riches, plus denses, ils rendent tout entier le son de la voix. Je tends, pour tout dire, à cette objectivité sans laquelle aucune œuvre d'art n'est complète. II s'agit, ni plus ni moins, d'aller vers les autres, de les situer dans l'univers, d'apprendre à cerner l'humain qui est hors de moi. Tâche excessivement difficile pour l'égoïste que je suis. De cette tension quelque chose, peut-être, sortira.

J.-P. D. Asies: sous ce titre à la Saint-John Perse, on trouve de brefs poèmes, d'allure très orientale. D'où ce recueil vous est-il venu? pourquoi avoir adopté cette forme particulière d'écriture poétique?

J. É.-B. J'avais d'abord voulu en faire un hommage à la Chine et à mon père, à l'Orient et à l'Occident. Le titre m'en avait paru ronflant, j'ai préféré Asies qui dit bien ce qu'il veut dire: que chacun de nous porte en lui les multiples mirages du monde. Est venu se greffer là-dessus ce mirage essentiel qui a nom amour. Recueil baroque. Je constate que j'ai hésité à y développer les thèmes qui lui conféraient son originalité. J'ai eu peur d'aller trop loin et de me tromper. J'ai eu peur de déformer le sens de mon expérience de l'Asie en la réduisant à une série de tableaux vivants. J'ai eu peur d'exprimer un amour fou. Je me trouvais pour la première fois en face de vrais problèmes d'écrivain et je n'ai pas su les résoudre. Asies a donc paru à l'état encore en quelque 
sorte d'ébauche. Une pierre au bord de ma route. Mais peut-être une pierre blanche. Qui sait? La qualité première de ma tentative en poésie, devant l'avenir, est qu'elle n'est en rien liée aux idées et aux sentiments transitoires de la littérature d'aujourd'hui. Je ne parle ni du «pays " ni de "l'aliénation", ni de l'esclavage historique. Tout cela passera. Peut-être est-ce déjà passé. L'ardeur politique est l'ennemie du style et il faut le génie d'un Stendhal ou d'un Soljénitsyne pour que de ce mariage de raison naisse l'amour. Aragon lui-même se dresse, au milieu de ses palinodies, comme le tenant d'un style. C'est par le style qu'il a commencé, c'est à lui qu'il est revenu. Les écrivains québécois ne se rendent pas compte que leur modernité, c'est-à-dire leur acceptation de l'inculture, les condamne à disparaître en moins d'une génération. Où sont nos génies d'il y a un quart de siècle? La littérature, nous l'avons toujours considérée comme un tremplin; de la terre de glaise au bureau directorial! Les exemples sont nombreux, qui ne débouchent sur aucune ceuvre.

Il est facile (et dangereux) de clamer en vers faciles son attachement à un pays dont on accélère le processus de décomposition par la faiblesse de son écriture. La poésie est autre chose. Fargue l'a dit, c'est une haute solitude. C'est aussi, à chaque instant, descente orphique en soi. Peu à peu, le poète accepte, à l'intérieur de son rythme, d'être l'homme qu'il est. Dieu seul sait à quel point il est difficile d'être soimême. L'acte poétique est le moyen le plus complexe d'apprendre à se connaître puisque chaque expérience de poésie dévoile l'existence en soi du néant. Est-ce être pessimiste que de constater que l'homme n'est rien? L'homme devient écrivain pour abolir cette loi. Nietzsche pense que "le plus haut sentiment de puissance et d'assurance s'exprime dans toute cuvre de grand style". Au centre de l'écriture, on trouve la passion apollinienne de connaître, de devenir partie d'universel. En Asie, j'ai appris à accepter que tout était relatif. Les grandes amours qu'on chante aujourd'hui et dont on croit qu'on va mourir, demain on se félicitera d'en avoir conçu les limites. Les poèmes que j'ai écrits sont peu faits pour plaire. Ils sont à la fois trop voluptueux et trop secs. Chacun, en les lisant, se demande à qui je m'adresse; à personne, sinon à la volupté et à la sécheresse mêmes. Mais, dès que j'ai commencé à écrire, je me suis dit que jamais je n'écrirais pour un lecteur hypothétique; son ombre s'étendrait sur ma pensée et je ne me sentirais plus libre. Je porte déjà en moi trop d'interdits pour que vienne, de mon propre chef, s'y ajouter une autre muraille de Chine, la moins infranchissable de toutes: le lecteur qui vous juge avant même que vous ayez offert à la page blanche ses premiers caractères magiques. Je me fie au langage et à lui seul. II me guide chaque fois que je m'exprime, c'est lui qui m'apprend, au fur et à mesure, ce que je veux dire; il me livre mon rythme, il m'apprend à respirer en fonction des mots, il me permet d'entrer dans des familles de vocables et d'y vivre à l'aise. J'ai aussi appris que la confiance ne peut s'établir entre un écrivain et son langage que dans la mesure où le premier a des égards pour le second. 
La recherche dans l'expression, la pratique du dictionnaire (qui doit être une lecture presque quotidienne) et celle, constante, d'un bon auteur, nécessités lorsqu'on écrit. On a toujours moins à dire qu'on ne pense. Ces riens, ces broutilles, il faut les exprimer aussi parfaitement que faire se peut. La postérité a toujours fait son choix en fonction de l'intensité du rapport entre un écrivain et son style. L'imposture, c'est de mal écrire; le fond est dans la forme.

J.-P. D. II y a deux noms qui signifient beaucoup pour vous, dans l'histoire de la pensée au Québec: François Hertel et l'abbé Groulx. A travers la vie et l'œuvre de ces deux penseurs, n'est-ce pas le devenir de l'homme québécois qui vous passionne, qui vous retient?

J. É-B. J'ai vécu ma jeunesse à l'ombre de la pensée de l'abbé Groulx. Je ne suis pas le seul. L'abbé Groulx était un homme fait pour inspirer le respect, susciter l'admiration et l'amour. Sa personnalité était mythique. Cela, nous le sentions obscurément. II avait des amis, certes, mais on le savait entouré, chez les historiens et les hommes politiques, d'ennemis. Tout ce qui souhaitait nous interdire la fierté d'être canadiensfrançais l'avait choisi comme cible. On diminuait son mérite d'historien; on lui faisait grief d'être un polémiste, d'imposer à l'histoire du passé une portée contemporaine. On lui reprochait surtout de travailler, de créer de toutes pièces, un corpus historique d'où n'était pas ábsente la littérature. On l'accusait, bien sûr, de confondre les genres. Les historiens, en général, n'aiment pas qu'un des leurs puisse s'élever audessus de ses fiches. L'abbé Groulx faisait des siennes de merveilleux messagers d'espoir. Son nationalisme canadien-français le desservait auprès des politiciens et des historiens canadiens-anglais nationalistes, car le nationalisme canadien-anglais ne souffre pas de contrepartie à ses côtés. A nos yeux de jeunes hommes, la grandeur de l'abbé Groulx résidait précisément dans cette lutte qu'avant quiconque au $x x^{\theta}$ siècle, il a menée afin de maintenir notre nation dans son être. II assurait, de plus, la transition entre le $X I X^{e}$ siècle (dont nous ignorons presque tout) et notre temps. Malgré leur devise, les Québécois n'ont pas le sens de la continuité. En général, ils ne se souviennent que de balivernes, Jos Montferrand ou la Corriveau, hissés sur le pavois du folklore. Du déroulement de ces luttes idéologiques qui nous ont menés où nous sommes, rien. La conscience de l'abbé Groulx étant historique de nature. il a tendu ses filets le plus loin possible afin que rien de nos tribulations et de nos espoirs ne puisse échapper à son analyse. C'est ainsi que, par l'esprit, il a repris possession de cette Amérique qui fut jadis française et qu'il a raconté notre essor missionnaire (au cours du dernier siècle, le seul moyen pour des milliers de Québécois, d'échapper aux contraintes intellectuelles et à la vulgarité sordide de notre milieu).

Sans modestie mensongère, l'abbé Groulx fut toujours un intellectuel. Le mot est galvaudé et Henri Bourassa s'en moquait déjà avec stridence au début du siècle. Grâce à la puissance et à la continuité de son travail créateur, l'abbé Groulx a redonné à ce mot son intensité et sa cha- 
leur. Écrivain, il a écrit. Ses Mémoires soulignent à chaque page à quel point il a refusé de se mentir à lui-même. Bon, il avait la dent dure. La bêtise n'était pas, de toute évidence, son fort. II a dû beaucoup souffrir. Homme de la ligne droite, il a vécu au milieu des tergiversations et des trahisons d'un milieu politique trop avili pour se reconnaître tel. Au milieu de ce déchet, l'abbé Groulx est resté prêtre et honnête homme. Il a continué à penser froidement, laissant son esprit comprendre I'histoire et son langage l'expliquer. Quiconque a eu l'honneur de le fréquenter, se souviendra de sa façon d'être, de son rire, de son agréable ironie, de l'étendue de ses connaissances, de ses mains, solides de paysan, sensibles d'écrivain.

Cet homme que j'admirais au collège, je l'ai connu à.l'Université de Montréal, où j'ai suivi ses cours. Ce fut, à la faculté des Lettres, sous l'égide de $M$. Jean Houpert, une époque faste. L'abbé Groulx m'apprit à ne pas me perdre dans la forêt des idées et $M$. Jean Houpert m'envoya rue d'Ulm. Dans une vie, ce sont, en effet, des rencontres fastueuses. Plus tard, avec son sourire, l'abbé Groulx me tint sur mes idées et l'innervation de mon écriture, des propos flatteurs. Comment ne pas s'incliner dans la joie devant le décret d'un homme dont le goût avait toujours été de haut parage?

J'admirais trop l'abbé Groulx, j'avais de lui une crainte trop révérencielle pour oser l'approcher de mon propre chef. L'âge venu, aucun grand homme n'échappe à la condescendance. L'écart entre l'abbé Groulx et moi était trop sensible pour que, de maître à élève, nous devinssions amis. A l'époque où je l'ai connu, l'abbé Groulx était déjà entré dans l'histoire, la tête haute. J'étais jeune homme fait.

C'est à la faveur du système d'enseignement des jésuites que nous nous sommes connus, Hertel et moi. II m'a aidé à traverser l'empêtrement tragique de mes quinze ans et m'accepte aujourd'hui dans la solitude de ma cinquantaine bien sonnée. II est difficile de savoir ce qu'on aime chez un être et Montaigne avec son "parce que " avait raison. Le secret du charme si puissant de Hertel, c'est qu'il ne cherche jamais à être un maître, à apprendre à penser, à sarcler les idées des autres. On oublie son rôle d'avant la dernière guerre. (On a oublié la dernière guerre.) Hertel fut un éveilleur. A coups de hache, il a débroussaillé les recoins de notre conscience collective. $A$ une génération d'hommes, il a appris à lire. Je revois le recteur de mon collège m'arrachant des mains le Soulier de satin et me menaçant des pires sanctions s'il me reprenait en flagrant délit. Hertel m'avait prêté ce livre. En Claudel le Père recteur déplorait le chantre des amours ardentes, moi la grande gueule et l'absence bovine de tout humour. Hertel brisait les murs de notre autarcie intellectuelle. Il y avait en lui un peu trop du philosophe et il préférait les écrivains qui font réfléchir. Lui-même aimait innover. II a écrit un livre de philosophie qui n'était pas thomiste. A l'époque, c'était une gageure. II fut l'un des premiers à concevoir une refonte globale du Canada politique et géographique. L'avenir retiendra peut- 
être sa solution, qui est originale. Dans notre poésie, le ton de la sienne est neuf. On y trouve un dialogue terrible avec Dieu, terrible parce qu'indécis. Hertel innova aussi en quittant les ordres. II le fit dans la dignité et ne devint pas danseur mondain. Son exemple a été suivi de la plus déplorable façon et ce qui, chez lui, relevait de la tragédie se dilua en farce chez les autres.

Sa personnalité ? Mélange de poète, de philosophe, de créateur de personnages qui sont des reflets multiples de son moi; l'inspirateur est en lui partout présent et il a consacré à conseiller des amis de précieuses heures arrachées à son travail. J'aime ses pirouettes, sa façon à la fois bon enfant et altière de parler de son œuvre et de sa vie, j'aime son rire tragique et l'insatisfaction métaphysique qui loge dans son regard. J'aime son enthousiasme physique. Aujourd'hui une maladie sourde l'emporte sur le dynamisme du skieur et du patineur, sur l'envol du joueur de crosse et de pelote basque. Mais dès qu'il s'anime, que le regard brille, qu'intervient le rire, la verve physique renaît et abolit le temps. Hertel est un être de solitude, à condition d'être entouré de livres, de l'affection (même lointaine) d'amis chers, d'avoir du travail, une table, des cigarettes. II se replie sur son aire et tient sa nervosité bien en main. Petit de taille, tassé, musclé, il s'agite comme un animal prêt à s'enfuir. On sent qu'il y a en lui des ressources inépuisables de renouvellement. Heureux dans sa chambre, il rêve aussi de grands départs. De là ses voyages. II faut l'avoir entendu raconter son voyage au Japon, sa rencontre avec Kyoto. Son regard redevient neuf. II est pur.

Mais, comme tout écrivain, c'est dans ses livres qu'on le trouve: imagination, énergie, conception optimiste et tragique tout ensemble de la vie, recours constant à l'intelligence sous sa forme la plus subtile qui a nom ironie socratique, style affirmatif, et, sous-jacent, le dialogue que Hertel entretient avec l'invisible. On le jugera sur son cuvre et non sur le propos de ses amis. Son œuvre ne le trahira pas. Elle est importante et servira de point de repère lorsqu'il s'agira de comprendre l'évolution intellectuelle du Canada français au $X X^{e}$ siècle. Hertel, par sa façon d'être se situe en marge; par sa pensée, il est au centre de notre comportement. On peut penser que l'homme inspirera les biographes; l'écrivain jettera sur son temps des lueurs qui éblouiront. Aujourd'hui, malheureusement, Hertel est dans la pénombre. D'abord, il habite Paris et ce qu'on pardonne volontiers à Anne Hébert, on ne le souffre pas chez Hertel. Il est exilé. Ensuite, ses ouvrages de pure virtuosité créatrice sont à la fois trop funambulesques et trop riches de culture pour plaire à une génération de lecteurs formés à la chansonnette poéticonationaliste et aux contrepèteries de $M$. Réjean Ducharme. II y a incompatibilité. Enfin, Hertel a un sens de l'humour et du ridicule qui se situe à l'extrême opposé de la vague moralisatrice qui sévit au Québec. Pour tout dire, Hertel a toujours été en avance sur son temps. Peut-être, un jour, les Québécois le rattraperont-ils.

(Entrevue réalisée par Jean-Pierre Duquette, Université McGill) 